\title{
Modeling Cerebral Blood Flow Control During Posture Change from Sitting to Standing
}

\author{
Mette Olufsen (msolufse@math.ncsu.edu) ${ }^{1}$ and Hien Tran (tran@ math.ncsu.edu) ${ }^{2}$ \\ Center for Research in Scientific Computation \\ Department of Mathematics \\ North Carolina State University \\ Raleigh, NC 27695-8205 \\ Johnny Ottesen (johnny@ ruc.dk) \\ Department of Mathematics and Physics \\ Roskilde University \\ Denmark
}

${ }^{1}$ The work of this author was supported in part by the National Institute of Health under grant RO3AG20833
${ }^{2}$ The work of this author was supported in part by the National Institute of Health under grant 1 RO1 GM67299-01 


\begin{abstract}
Hypertension, decreased cerebral blood flow, and diminished cerebral blood flow regulation, are among the first signs indicating the presence of cerebral vascular disease. In this paper, we will present a mathematical model that can predict blood flow and pressure during posture change from sitting to standing. The mathematical model uses a compartmental approach to describe pulsatile blood flow and pressure in a number of compartments representing the systemic circulation. Our model includes compartments representing the trunk and upper extremities, the lower extremities, the brain, and the heart. We use physiologically based control mechanisms to describe the regulation of cerebral blood velocity and arterial pressure in response to orthostatic hypotension resulting from postural change. To justify the fidelity of our mathematical model and control mechanisms development, we will show validation results of our model against experimental data from a young subject.
\end{abstract}

\title{
1 Introduction
}

The understanding of short term cardiovascular regulation of blood flow to the brain is essential for development of new strategies to prevent cognitive loss, falls, and syncope, which are major causes of morbidity and mortality in elderly people. In this paper, we focus our study on short term cardiovascular regulation by analyzing arterial blood pressure and cerebral blood flow velocity during postural change from sitting to standing. The most important short term regulatory mechanisms are autonomic reflexes and cerebral autoregulation. Autonomic reflexes are mediated via sympathetic and parasympathetic nervous responses while cerebral autoregulation is a local control mediated via changes in active tone in the small blood vessels in the brain. Previous studies have shown that one of the most important autonomic reflexes, the arterial baroreflex, responds to postural change by inducing cardiac acceleration and peripheral vasoconstriction, while cerebral autoregulation regulates cerebral blood flow velocity through cerebral vasodilation $[10,17]$. The interaction between the two types of regulation is not well understood. It is known that with aging, regulatory capacity declines, and it is believed that the cardiovascular regulation is impaired in cardiovascular diseases such as hypertension [3, 26, 29].

The overall goal of this paper is to use advanced methods of mathematical modeling to understand the interaction between autonomic reflexes and cerebral autoregulation during postural change from sitting to standing. Specific attention will be paid to incorporating parameters repre- 
senting various physiological/biological mechanisms that can be validated using clinical data. To study these effects we build upon our previous work which suggested (using a 3-element windkessel model) that both systemic and cerebrovascular resistances play a major role in cerebral blood flow regulation during postural change from sitting to standing [17]. To explore this further, we have developed a closed loop compartmental model comprising the heart and the systemic circulation [5, 20,21]. This model includes compartments that represent the heart, the aorta, arteries in the brain, trunk/upper extremities, and the lower extremities, and the corresponding veins. Having a closed loop model enables us to directly model the regulation and then fit the model to data to determine if our model adequately fits the data. Before fitting the data to the model initial values for all parameters are computed using standard values of pressures and volume distributions for an average person. Then, the model was fit to the data to obtain specific values for the subject studied in the current paper.

The data we used for validating the compartment model include physiological recordings of blood pressure and blood flow velocity. Blood flow velocity was measured in the middle cerebral artery (MCA) using transcranial Doppler (TCD) methods. Blood pressure was measured in the index finger using a Finapres cuff (see Figure 1). The measurements used for validation in this paper are recordings from one subject during postural change from sitting to standing. After instrumentation and after a steady signal was obtained, blood flow velocity and pressure was measured for 60 seconds before the subject stood up. After standing the measurements were continued for another $60 \mathrm{sec}$.

The organization of the paper is as follows. In section 2 , we give a brief overview on the physiology of blood flow and pressure regulation. This summary is necessary for the development of the mathematical model in section 3. Section 4 contains our results and discussions.

\section{Blood Flow and Pressure Regulation - The Physiology}

Short-term cardiovascular regulatory mechanisms are intended to buffer transient changes in blood flow to vital organs occurring as a consequence of posture change, exercise, hemorrhage, or other 
acute stresses. The buffering is accomplished by two mechanisms: (a) Autoregulatory adaption of blood vessels to altered or redistributed volumes and pressure (b) rapid autonomic sympathetic and parasympathetic neural responses affecting the heart rate, cardiac contractility, and vascular resistance [8].

Autonomic reflexes are mainly mediated via baroreflexes. Baroreceptors are stretch receptors which are sensitive to pressure alterations. High pressure receptors are located in the aortic arch and carotid sinuses and low pressure receptors are thought to be located in the atria and pulmonary circulation. The receptors act in concert in an effort to buffer arterial blood pressure changes. They have cardiovascular and renal manifestations and they can adapt to a sustained alteration by resetting. In this paper, we focus on hypotensive changes occurring during postural change from sitting to standing, and hence, we have focussed on modeling autonomic reflexes and cerebral autoregulation $[8,25]$.

During postural change from sitting to standing, approximately $500 \mathrm{cc}$ of blood is pooled in the legs as the result of gravitational force [11]. As a consequence, the blood pressures in the trunk and upper extremities drop. To compensate for this drop in pressure, short term regulation mechanisms are activated. Autonomic reflexes work in response to the decreased arterial blood pressure by activating the sympathetic system and deactivating the parasympathetic system. The increase in sympathetic activity will restore the blood pressure to normal due to an increase in heart rate, myocardial contractility, vasoconstrictor, and venoconstrictor tone. The sympathetic nervous system normally responds to a decrease in blood pressure rather than an increase (i.e., it is more effective in combating acute hypotension than acute hypertension). The control forms a hysteresis effect, an increased sympathetic activity is typically initiated within seconds of the stimulus and it is able to increase the pressure within 5-10 seconds, whereas sudden inhibition of nervous stimulation requires 10-40 sec to decrease the arterial blood pressure. In addition to the increased sympathetic activity, a parasympathetic withdrawal helps to restore the blood pressure. Parasympathetic withdrawal mainly increases the heart rate, it has a negligible effect on the cardiac contractility and almost no effect on the peripheral resistance. The parasympathetic response is very fast, faster 
than the sympathetic response. Its effect will be implemented within 1-2 cardiac cycles. In addition, the parasympathetic response is greatest in the normal range of blood pressure (from 80-150 $\mathrm{mmHg})[8,25]$. However, the baroreceptors are able to adapt to permanent or semipermanent alterations in arterial blood pressure. Even in acute hypertension (6 hours or less) and in exercise, the receptors continue to function but are reset at a higher level, which then becomes the baseline for further response. This reset is reversible if the baseline pressure changes. Experimental studies have shown that with age the rapidity and the level of the reflex responses take longer. It has been shown that in the seventh decade the magnitude of the response is decreased up to $50 \%[3,12]$.

Autoregulation is a local control mechanism that regulates blood flow in proportion to the metabolic needs of the tissue. The aim of autoregulation is to keep the blood flow constant within some range of mean arterial blood pressure (from $80-150 \mathrm{mmHg}$ ). To obtain a constant blood flow, a drop in pressure is compensated by vasodilation of arterioles and an increase in pressure by arteriolar vasoconstriction. Outside this range, changes in flow will follow changes in pressure. When the pressure becomes too low the vessels are already dilated to their maximum and as a result the flow will drop. When the pressure becomes too high, vasoconstriction is overcome by the pressure leading to hyperemia. It should be noted that the ranges listed above are for a normal mean blood pressure of approximately $100 \mathrm{mmHg}$. This range can be reset to operate at higher or lower pressures as the result of long term effects such as chronic hypertension. Autoregulation is mediated via changes of active tone in the small blood vessels. It is not clear exactly how this change is mediated. It is believed that two mechanisms, myogenic responses and oxygen demand responses, are engaged $[3,13,23,28]$. The myogenic responses reacts to an increased perfusion pressure by increasing muscle tension stimulating the arterioles to contract to reduce flow $[23,28]$. Oxygen demand responses reacts to a decrease in flow producing a decrease in tissue $\mathrm{O}_{2}$ concentration, which yields an increase in concentration of $\mathrm{CO}_{2}$ and other metabolites $[2,3,4]$. As a result, the arterioles relax and the flow rises again. The physiological responses in either of the two cases involve local blood flow regulation mediated via negative feedback mechanisms. For healthy young people autoregulation is typically delayed by 5-10 seconds from when the change 
in flow was initiated. Autoregulation takes place in the brain and in all major organs. To study fast regulatory responses during postural change from sitting to standing, we plan to study effects of cerebral autoregulation (CA), and neglect regulation in other organs that occurs over longer timescales.

\section{Methods}

\subsection{Experimental Measurements}

The experimental study included ten young carefully screened healthy young volunteers aged 2039 years. In this work, we studied data from one subject. The mean cerebral autoregulatory responses to posture change and carbon dioxide levels are described elsewhere $[10,17]$.

During the protocol, heart rate was measured continuously from a 3-lead electrocardiogram and beat-to-beat arterial pressure was determined non-invasively from the middle finger of the nondominant hand, using a photoplethysmographic non-invasive Finapres pressure monitor (Ohmeda Monitoring Systems, Englewood, CO), supported by a sling at the level of the right atrium to eliminate hydrostatic pressure effects. In order to keep end-tidal $\mathrm{CO}_{2}$ constant, respiration was measured continuously using an inductive plethysmograph (Respitrace, Ambulatory Monitoring, Ardsley, NY) and subjects breathed at $0.25 \mathrm{~Hz}$ (15 breaths per minute) throughout each standing procedure by following tape-recorded cues. All subjects underwent Doppler ultrasonography by a trained technician in order to measure the changes in blood flow velocity within the MCA in response to active standing. The $2 \mathrm{MHz}$ probe of a portable Doppler system was strapped over the temporal bone and locked in position with a Mueller-Moll probe fixation device to image the MCA DWL Doppler system (MultiDop X4, DWL-Transcranial Doppler Systems Inc., Sterling, VA). The MCA blood flow velocity was identified according to the criteria of Aaslid [1] and recorded at a depth of 50-65 mm. The envelope of the blood flow velocity waveform, derived from a FastFourier analysis of the Doppler frequency signal, and continuous pressure and EKG signals were digitized at $250 \mathrm{~Hz}$ and stored in the computer for later off-line analysis.

Following instrumentation, subjects sat in a straight-backed chair with their legs elevated at 90 
degrees in front of them on a stool. For each of two active stands, subjects rested in the sitting position for 5 minutes, then stood upright for one minute. The initiation of standing was timed from the moment both feet touched the floor. Data were collected continuously during the final minute of sitting and the first minute of standing during both trials. Data to be analyzed in this paper include the last minute of sitting and the one minute of standing.

The study was approved by the Institutional Review Board at the Hebrew Rehabilitation Center for Aged, and all subjects provided written informed consent. In addition, data was used by permission from Dr. Lipsitz at the Hebrew Rehabilitation Center for Aged, Boston, Massachusetts.

\subsection{Mathematical Model}

To predict blood pressure in the finger and flow velocity in the MCA we have developed a compartment model of the systemic circulation based on volume conservation laws [30]. In our model, the systemic circulation is divided into 6 compliant compartments that represent the arteries and veins and one compartment that represent the left ventricle (see Figure 2). Three compartments represent the systemic arteries (the brain, the lower body (legs), and the upper body) and three similar compartments represent the systemic veins. The design of the systemic circulation with arteries and veins separated by capillaries provides some resistance and inertia to the volumetric blood flow rate. In our model we include effects of resistance between compartments but neglect effects due to inertia. The description of pressure and volumetric flow rate in a system comprised of compliant compartments (capacitors) and resistors can be interpreted in terms of an electrical circuit, where pressure $p[\mathrm{mmHg}]$ plays the role of voltage and volumetric flow rate $q\left[\mathrm{~cm}^{3} / \mathrm{sec}\right]$ plays the role of current. Note that the blood flow velocity measured in the MCA can be obtained by dividing the volumetric flow rate with the area of the vessel. In the remainder of this section we will use the term "flow $q$ " to describe the volumetric flow rate.

The basic equations predicting blood pressure and flow can be obtained by computing the volume and change of volume for each compartment. The equations representing the arterial and venous compartments can all be described similarly. For each of these compartments the volume 
$V=C p\left[\mathrm{~cm}^{3}\right]$, where $C\left[\mathrm{~cm}^{3} / \mathrm{mmHg}\right]$ is the compliance and $p[\mathrm{mmHg}]$ is the pressure. The cardiac output from the heart is given by $V=V_{\text {stroke }} H$, where $V_{\text {stroke }}$ is the stroke volume and $H$ [beats/sec] is the heart rate. For each compartment, the net change of volume is given by the flow into the compartment minus the flow out of the compartment;

$$
\frac{d V_{i}}{d t}=q_{\text {in }}-q_{\text {out }} .
$$

For the compartment representing the upper arteries there are two flows leaving the compartment $\left(q_{\text {out }}=q_{\text {aup }}+q_{a l}\right)$ and for the compartment representing the upper veins there are two flows entering the compartment $\left(q_{\text {in }}=q_{a u p}+q_{v l}\right)$. All other compartments have one inflow and one outflow. For the heart it is necessary to take the state of the heart valves into account. The model discussed in this paper only includes the left ventricle, hence, the states of the mitral and the aortic valves must be taken into account. During diastole the mitral valve is open (op) while the aortic valve is closed (cl) allowing blood to enter the ventricle, when the ventricle is full the ventricle contracts to increase the pressure. Once the ventricular pressure is above the aortic pressure, the aortic valve opens and a pulse-wave is pumped out. It is noted that the state where both valves are open does not occur under normal condition. Consequently, the change in volume of the left ventricle can be modeled as:

$$
\frac{d V_{l}}{d t}=\left\{\begin{array}{cl}
q_{m v} & \text { mv-op, av-cl } \\
q_{m v}-q_{a v} & \text { mv-op, av-op } \\
0 & \text { mv-cl, av-cl } \\
q_{a v} & \text { mv-cl, av-op }
\end{array}=s_{m v} q_{m v}-s_{a v} q_{a v}\right.
$$

where $s_{m v}=1$ if the mitral valve is open and 0 otherwise and $s_{a v}=1$ if the aotic valve is open and zero otherwise. With this state variable added, the equation (2) has the same functional form as (1). Conservation of volume $V$ requires that $V_{t o t}=\sum_{i} V_{i}$ is constant or that $d V_{t o t} / d t=0$.

The equations derived above provide relations between volume and flow and volume and pressure. To obtain relations between flow and pressure an additional equations must be derived. Kirchoff's current law provides a linear relation between pressure and flow of the form:

$$
q_{i}=\frac{p_{\text {in }}-p_{\text {out }}}{R_{i}}
$$


where $R_{i}$ is the resistance to the flow. Differentiating the volume equation $V_{i}=C_{i} p_{i}$ and inserting (1) gives

$$
C_{i} \frac{d p_{i}}{d t}=q_{\text {in }}-q_{\text {out }}-p_{i} \frac{d C_{i}}{d t}
$$

The term containing the derivative of $C_{i}$ is located on the right hand side since we assume that a functional relation can be obtained for the compliance. The circuit in Figure 2 will give rise to a total of 6 differential equations of the form (4), one for each of the arterial and venous compartments. To model the branching before and after the left ventricle two algebraic equations for $p_{a v}$ and $p_{m v}$ are computed. These can be obtained by ensuring conservation of flow $\left(q_{a v}=q_{a c}+q_{a u}\right.$ and $\left.q_{m v}=q_{v u}+q_{v c}\right)$.

The only pressure not yet defined in terms of an equation is the left ventricular pressure. To obtain a pulse-wave that is propagated along the artery this pressure must be described in terms of an activation function. There are a number of such functions defined in the literature [14, 22, 27]. In the current work, we will use the model by Ottesen [22] since it provides the ability to use a variable heart-rate as well as specifying both the onset of the ventricular contraction and relaxation. This model is given by

$$
p_{l}=a\left(V_{l}-b\right)^{2}+\left(c V_{l}-d\right) g(t),
$$

where $p_{l}$ is an isovolumic pressure that is modeled using the parameters $a, b, c$, and $d$, and an activation function $g(t)$. The parameter $a\left[\mathrm{mmHg} / \mathrm{cm}^{3}\right]$ relates to ventricular elastance during relaxation and $b\left[\mathrm{~cm}^{3}\right]$ represents ventricular volume for zero diastolic pressure, the parameters $c$ $\left[\mathrm{mmHg} / \mathrm{cm}^{3}\right]$ (contractility) and $d$ relate to the volume dependent and volume independent components of the developed pressure $[7,22]$. The activation is described by a polynomial of degree $(n, m)$ and it provides an expression for $g(t)=f(t) / f\left(t_{p}\right)$ with

$$
f(t)= \begin{cases}0, & 0 \leq t<\alpha \\ p_{p}(H) \frac{(t-\alpha)^{n}(\beta(H)-t)^{m}}{n^{n} m^{m}[(\beta(H)-\alpha) /(m+n)]^{m+n}}, & \alpha \leq t \leq \beta(H) \\ 0, & \beta(H)<t \leq T\end{cases}
$$

where $T$ [sec] is the length of the cardiac cycle, $H$ is the heart-rate, the parameters $\alpha$ and $\beta(H)$ 
[sec] denote the onset of contraction and relaxation, respectively. The parameters $n$ and $m$ characterize the contraction and relaxation phases of the left ventricle, and the parameter $p_{p}$ is the peak value of the activation function. Note that the polynomial function has compact support with a simple algebraic expression. The ability to vary the heart-rate is included in the isovolumic pressure equation (5) by scaling time and peak values of the activation function $f$. This is possible since the compact support causes the ventricular end of contraction $\beta$ to appear explicitly in (6). The time for peak value of the contraction is scaled by introducing a sigmoidal function between time for peak pressure $t_{p}$ and heart-rate $H$ of the form:

$$
t_{p}=t_{\min }+\frac{\theta^{\nu}}{H^{\nu}+\theta^{\nu}}\left(t_{\max }-t_{\min }\right)
$$

where $\theta$ represents the median and $\nu$ represents the steepness of the relation, $t_{\min }$ and $t_{\max }$ denote the minimum and maximum values, respectively. The peak ventricular pressure $p_{p}$ is scaled similarly using a sigmoidal function of the form:

$$
p_{p}=p_{\min }+\frac{H^{\eta}}{H^{\eta}+\phi^{\eta}}\left(p_{\max }-p_{\min }\right),
$$

where $\phi$ represents the median and $\eta$ represents the steepness of the relation, $p_{\min }$ and $p_{\max }$ denote the minimum and maximum values, respectively.

Finally, the time for onset of ventricular relaxation $\beta(H)$ is modeled as

$$
\beta(H)=\frac{n+m}{n} t_{p}(H)-\frac{\alpha m}{n} .
$$

This equation is obtained by recognizing that the time for peak pressure $t_{p}$ is related to the parameter $\beta$ in the isovolumic pressure model (5). Initial values for all parameters were obtained from the work by Ottesen and Danielsen [22]. In their work, the parameters were obtained by validating their model to data from a dog. To obtain values that would work for a person, we fitted the parameters during our model validation. The resulting parameters can be found in Table 1 .

We have modeled transition from sitting to standing by including gravitational forces to the pressures in the lower compartments $a l$ and $v l$ as:

$$
\left[p_{a l}\right]_{s t}=\left[p_{a l}\right]_{s i t}+\rho g h(t), \quad\left[p_{v l}\right]_{s t}=\left[p_{v l}\right]_{s i t}+\rho g h(t),
$$


where $\rho=1.055\left[\mathrm{~g} / \mathrm{cm}^{3}\right]$ is the density, $g=981\left[\mathrm{~cm} / \mathrm{s}^{2}\right]$ is the gravitational acceleration, and $h[\mathrm{~cm}]$ is the change in height from sitting to standing. To capture the transition from sitting to standing $h$ is defined as the stepwise increasing linear function

$$
h(t)= \begin{cases}0, & t<t_{s t} \\ h_{M}\left(t-t_{s t}\right) / \tau, & t_{s t} \leq t \leq t_{s t}+\tau \\ h_{M}, & t \geq t_{s t}+\tau\end{cases}
$$

where $t_{s t}$ is the time at which the subject stands up, $\tau$ is the duration of the transition, and $h_{M}$ is the maximum height needed for the mean arterial pressure in the finger to drop as indicated by the data. The parameters for this equation have to be justified based on measurements of displaced volumes and observed pressure changes. Results from fitting the model to data from one subject gave that $h_{M}=25.26[\mathrm{~cm}]$ and $t_{s t}=1.56[\mathrm{sec}]$, see Table 3 .

To include regulatory compensation (autonomic and autoregulation) responding to restore blood pressure and flow, heart-rate, contractility, resistors, and capacitors are controlled. The control will be implemented by defining the model parameters (resistors, capacitors, heart-rate, and contractility) as functions of time [21].

The autonomic regulation is modeled as a pressure regulation where heart-rate $(H)$, cardiac contractility (c), systemic resistances $\left(R_{a u}, R_{a l}, R_{a u p}, R_{a l p}, R_{a c}\right)$, and systemic compliances $\left(C_{a u}\right.$, $\left.C_{a l}, C_{a c}, C_{v l}, C_{v u}, C_{v c}\right)$ are functions of the arterial pressure $p_{a u}$. The autoregulation affecting especially the cerebrovascular resistance is modeled using optimal control. In particular, we assume that $R_{a c p}$ is parameterized as follows:

$$
R_{a c p}(t)=\sum_{i=1}^{n} \gamma_{i} H_{i}(t),
$$

where $H_{i}$ are the standard Hat functions given by

$$
H_{i}(t)= \begin{cases}\frac{t-t_{i-1}}{t_{i}-t_{i-1}}, & t_{i-1} \leq t \leq t_{i} \\ \frac{t_{i+1}-t}{t_{i+1}-t_{i}}, & t_{i} \leq t \leq t_{i+1} \\ 0, & \text { otherwise }\end{cases}
$$


and the coefficients $\gamma_{i}$ are unknown to be estimated with other unknown parameters in the model via an inverse least squares problem.

The functional relations for the parameters have been obtained in two steps: The change in the controlled parameter is modeled using a first order differential equation with a set-point function dependent on the mean pressure $\bar{p}_{a u}$.

$$
\frac{d x(t)}{d t}=\frac{-x(t)+x_{c t r}\left(\bar{p}_{a u}\right)}{\tau} .
$$

Here, $x(t)$ is the controlled parameter and $x_{c t r}\left(\bar{p}_{a u}\right)$ is the set-point function, and $\tau$ is a time constant characterizing the time it takes for the controlled variable to obtain its full effect. Different values of $\tau$ were used for control of cardiac contractility, compliance, and resistances (see Table 3). The set-point function is given by

$$
x_{c t r}\left(\bar{p}_{a u}\right)=\left(x_{\max }-x_{\min }\right) \frac{\bar{\alpha}_{2}^{k}}{\bar{p}_{a u}^{k}+\bar{\alpha}_{2}^{k}}+x_{\text {min }} .
$$

The control equation is used for parameters that increase as a function of $\bar{p}_{a u}$. The parameters $x_{\max }$ and $x_{\min }$ are minimum and maximum values for the controlled parameter $x$ (we control $R_{a u p}, R_{a l p}, R_{a l}, R_{a u}, c$, and all compliances using this model), $\bar{\alpha}_{2}$ is the pressure at which we want to achieve the mean value $\left(x_{\max }+x_{\min }\right) / 2$, and $k$ provides the steepness of the sigmoid. The parameter $\bar{\alpha}_{2}$ is computed such that $x$ gives the right value of the controlled parameter $x$ at the steady state value $\bar{p}_{a u}$. As initial values of parameters for $k, x_{\min }$, and $x_{\text {max }}$, we used parameters suggested by Danielsen [6]. All of these parameters were fitted to data to obtain parameter values that were better suited for our study during postural change. To obtain values for $x_{\min }$ and $x_{\max }$ we fitted a factor $f$ such that $x_{\min }=x_{\text {set }} / f$ and $x_{\max }=x_{\text {set }} f$, where $x_{\text {set }}$ is the set-point value used during steady state (sitting). These set-point values can be found in Tables 1 and 2 . We did not fit the factor and steepness separately for all parameters but separated them into groups. In this way we had one set of parameters for $k$ and $f$ for arterial resistances $R_{a k}, R_{a f}$. Other groups included cardiac contractility $c_{k}$ and $c_{f}$, venous compliances $C_{v k}, C_{v f}$, arterial compliances $C_{a k}$, $C_{a f}$, cerebral venous compliances $C_{v c k}, C_{v c f}$, and cerebral arterial compliances $C_{a c k}, C_{a c f}$. Values for these parameters obtained after optimization can be found in Table 3. 
The above control equations are all based on values of the mean arterial pressure and the mean cerebral blood flow. However, our circuit model only describes the instantaneous (pulsatile) values. Mean values are computed as weighted averages where the present time is weighted more than past time:

$$
\bar{x}=\frac{1}{N} \int_{0}^{t} x(s) \exp (-\psi(t-s)) d s
$$

where $x=\bar{p}_{a u}$. The factor $\psi$ were found to be 0.002 from fitting our model to data, see Table 1 . The factor $N$ is a normalization factor to ensure that for $x(s)=1$ we get the correct mean pressure, i.e.,

$$
N=\int_{0}^{t} \exp (-\psi(t-s)) d s=\frac{1-\exp (-\psi t)}{\psi} .
$$

Differentiating (16) gives the following differential equation:

$$
\frac{d \bar{x}}{d t}=\frac{-\bar{x}+x(t)}{N}
$$

The system of differential equations from our mathematical model, (4), (14), and (18), are solved using MATLAB (The Math Works, Inc., Natick, MA) differential equations solver ode45. The initial time value for all numerical simulations corresponds to the time value of $50 \mathrm{sec}$ in the data. All unknown parameters are estimated from the data via a nonlinear inverse least squares problem. Values for all parameters and variables can be found in Tables 1 to 3.

\section{Results and Discussion}

In this section, we will discuss some of the preliminary results obtained with our mathematical model. The aim with our modeling efforts is to be able to reproduce changes in arterial blood pressure and cerebral blood flow during sitting to standing. Figure 3 shows the traces obtained from a young subject. The characteristic features are that after standing up at $60 \mathrm{sec}$, the pressure (both systolic and diastolic values) drops significantly. From a mean pressure of approximately 95 $\mathrm{mmHg}$ to a mean pressure of approximately $58 \mathrm{mmHg}$. At the same time, the blood flow velocity also decreases followed by an increase, however, it should be noticed that while the mean velocity 
decreases, the decrease is not as big, because a large widening of the pulse-amplitude (systolic value minus diastolic value). The aim with our model is to be able to reproduce the characteristics observed in the data discussed above.

The first step towards being able to reproduce the data is for our model to be able to reproduce the dynamics during steady state (i.e., during sitting). We obtained initial parameter values from physiological considerations for the distribution between the total blood volume between the compartments and initial estimates for the pressure values in the various compartments. The total amount of blood was computed as a function of the total body surface area, body weight, height, and sex [24]. The distribution of the total blood volume are obtained using the quantities suggested by [6]. After obtaining initial guesses of parameters, we fitted our model (without including equations describing the control (14)) to the datasets shown in Figure 3. To obtain the correct length for each cardiac cycle we extracted the heart-rate from the data, see Figure 4. Results of our simulation are depicted in Figure 5. The figure shows that without control we have obtained an excellent agreement between our model and the data. The model is fitted to the data using the Nelder-Mead algorithm, which is based on function information computed on sequences of simplexes [9].

The second step in validating our model is to illustrate that we can redistribute the volumes after standing up. The result of redistributing the volume is that both cerebral blood flow velocity and the arterial pressure is decreased. Again, we show the result without including the effects of the control. These results are shown in Figure 6. This figure indicates that with the parameters listed in Tables 1 to 3 it is possible to decrease both blood flow velocity and pressure. Two things should be noted about this figure: First, while we did not include effects of the control, we still see an increase in heart-rate, because heart-rate information is subtracted from the data (see Figure 4). Second, it should be noted that even though both blood flow velocity and pressure drops, the pulseamplitude for both blood flow velocity and pressure are too narrow. One reason for this could be that we need to investigate the role of compliances further. Compliance is related to the volume through $C p=V$.

Next, we will show simulation results obtained when all control mechanisms are activated. 
Figure 7 depicts the final result for both blood flow velocity and blood flow pressure and Figure 8 displays a close-up plot for $73.8 \leq t \leq 84.5$. The results show that even though our model does not quite capture the dynamics during the transitional region it is very good at obtaining the new steady state values during standing. However, it should be noticed that we are currently not able to capture the widening of the blood flow velocity during the transitional region. This may be partially explained from the result we got for the cerebrovascular resistance. This resistance is not obtained by a model, since its control includes effects both of autoregulation and of autonomic regulation. Instead we have used optimal control to fit the data to the model (see equation (12)). We have chosen to include nine points to represent the dynamics of the resistance $\left(\gamma_{1}\right.$ to $\gamma_{9}$; see Table 3). The result is shown in Figure 9. This figure shows a small initial increase followed by a decrease. From earlier work [17] we expected an increase, however much bigger than the one observed in our simulation. In fact the figure shows a decrease followed by an increase and the increase does not come above the base value. The rough results could be an artifact from only having the cerebral resistance represented by 9 points.

Finally, Figure 10 depicts the dynamics of some of the controlled variables. The figure shows one arterial resistance $R_{\text {aup }}$, the cardiac contractility $c$, and a venous compliance $C_{v u}$. These results do display quite different dynamics of the three types of variables. It should be noted that the dynamics of other resistances and capacitors are similar to the ones shown here. Most noticeable is it that the compliance does not seem to reach a steady state before we stand up. We believe that changes in compliance as well as changes in unstressed volumes are partly responsible for the fact that we did not completely capture the detailed dynamics during the transition from sitting to standing. Another important deficiency that we plan to study in the future, is that our model does not include specific time delays. Earlier work by Ottesen [19] has shown that time delays are important and that they can give rise to dynamics that cannot be captured without them.

In summary, we have developed a model in which the controls have been obtained by including biological mechanisms for changing resistors, cardiac contractility, and compliances. Our results show that our model is able to capture the increase in pressure and blood flow velocity needed to 
compensate for hypotension obtained during postural change from sitting to standing. However, more work is needed to capture the details of the regulation observed during the transitional period. 


\begin{tabular}{|c|c|c|c|}
\hline $\begin{array}{l}\text { Resistances } \\
{\left[\mathrm{mmHg} \mathrm{sec} / \mathrm{cm}^{3}\right]}\end{array}$ & & Heart parameters & \\
\hline$R_{a v}$ & 0.0004 & $a\left[\mathrm{mmHg} / \mathrm{cm}^{6}\right]$ & 0.0003 \\
\hline$R_{m v}$ & 0.0037 & $b\left[\mathrm{~cm}^{3}\right]$ & 6.3041 \\
\hline$R_{v l}$ & 0.1541 & $d[\mathrm{mmHg}]$ & 1.1264 \\
\hline$R_{v u}$ & 0.0302 & $n$ & 5.9717 \\
\hline$R_{v c}$ & 0.2159 & $m$ & 4.0917 \\
\hline$R_{a c}$ & 1.1355 & $f c t$ & 0.3614 \\
\hline & & $\nu$ & 9.1682 \\
\hline & & $\theta[$ beats/sec $]$ & 1.1364 \\
\hline & & $t_{\min }[\mathrm{sec}]$ & 0.0801 \\
\hline & & $t_{\max }[\mathrm{sec}]$ & 0.2653 \\
\hline & & $p_{\min }[\mathrm{mmHg}]$ & 1.0994 \\
\hline & & $p_{\max }[\mathrm{mmHg}]$ & 1.2028 \\
\hline & & $\eta$ & 19.0681 \\
\hline & & $\alpha[\mathrm{sec}]$ & 0.0000 \\
\hline & & $\phi[$ beats/sec $]$ & 1.3296 \\
\hline
\end{tabular}

Table 1: The values listed in this table are for uncontrolled parameters. Resistances are used in equation (3), and parameters for the heart are used in equations (5) and (6). 


\begin{tabular}{|l|r|}
\hline Setpoints & \\
\hline$R_{a u}\left[\mathrm{mmHg} \mathrm{sec} / \mathrm{cm}^{3}\right]$ & 0.0700 \\
$R_{a u p}\left[\mathrm{mmHg} \mathrm{sec} / \mathrm{cm}^{3}\right]$ & 2.6410 \\
$R_{a l p}\left[\mathrm{mmHg} \mathrm{sec} / \mathrm{cm}^{3}\right]$ & 3.1209 \\
$R_{a l}\left[\mathrm{mmHg} \mathrm{sec} / \mathrm{cm}^{3}\right]$ & 3.5657 \\
$C_{v u}\left[\mathrm{~cm}^{3} / \mathrm{mmHg}\right]$ & 14.1549 \\
$C_{v l}\left[\mathrm{~cm}^{3} / \mathrm{mmHg}\right]$ & 10.3195 \\
$C_{v c}\left[\mathrm{~cm}^{3} / \mathrm{mmHg}\right]$ & 7.8787 \\
$C_{a u}\left[\mathrm{~cm}^{3} / \mathrm{mmHg}\right]$ & 0.7382 \\
$C_{a l}\left[\mathrm{~cm}^{3} / \mathrm{mmHg}\right]$ & 1.2873 \\
$C_{a c}\left[\mathrm{~cm}^{3} / \mathrm{mmHg}\right]$ & 0.0279 \\
$c\left[\mathrm{mmHg} / \mathrm{cm}^{3}\right]$ & 2.2311 \\
$\bar{p}_{a u}[\mathrm{mmHg}]$ & 92.7000 \\
\hline
\end{tabular}

Table 2: Set-point values for all controlled parameters. These parameters are used to control maximum and minimum values for the controlled functions discussed in equation (14). 


\begin{tabular}{|l|r|l|r|}
\hline \multicolumn{4}{|c|}{ Optimized Parameters } \\
\hline$\tau_{S}[\mathrm{sec}]$ & 2.8890 & $R_{a k}\left[\mathrm{mmHg} \mathrm{sec} / \mathrm{cm}^{3}\right]$ & 1.5401 \\
$\tau_{C}[\mathrm{sec}]$ & 11.3246 & $R_{a f}\left[\mathrm{mmHg} \mathrm{sec} / \mathrm{cm}^{3}\right]$ & 7.0067 \\
$\tau_{R}[\mathrm{sec}]$ & 9.9907 & $C_{k}$ & 2.3516 \\
$h_{M}[\mathrm{~cm}]$ & 25.2647 & $c_{f}$ & 1.4609 \\
$t_{s t}[\mathrm{sec}]$ & 1.5628 & $C_{v k}$ & 26.2226 \\
$\gamma_{1}\left[\mathrm{mmHg} \mathrm{sec} / \mathrm{cm}^{3}\right]$ & 3.8734 & $C_{v f}$ & 11.4609 \\
$\gamma_{2}\left[\mathrm{mmHg} \mathrm{sec} / \mathrm{cm}^{3}\right]$ & 3.4381 & $C_{a k}$ & 14.2914 \\
$\gamma_{3}\left[\mathrm{mmHg} \mathrm{sec} / \mathrm{cm}^{3}\right]$ & 3.0587 & $C_{a f}$ & 3.8616 \\
$\gamma_{4}\left[\mathrm{mmHg} \mathrm{sec} / \mathrm{cm}^{3}\right]$ & 3.5190 & $C_{a c k}$ & 0.1025 \\
$\gamma_{5}\left[\mathrm{mmHg} \mathrm{sec} / \mathrm{cm}^{3}\right]$ & 3.2199 & $C_{a c f}$ & 4.3577 \\
$\gamma_{6}\left[\mathrm{mmHg} \mathrm{sec} / \mathrm{cm}^{3}\right]$ & 2.3538 & $C_{v c k}$ & 5.2542 \\
$\gamma_{7}\left[\mathrm{mmHg} \mathrm{sec} / \mathrm{cm}^{3}\right]$ & 3.6325 & $C_{v c f}$ & 14.7510 \\
$\gamma_{8}\left[\mathrm{mmHg} \mathrm{sec} / \mathrm{cm}^{3}\right]$ & 3.6450 & $\psi[1 / \mathrm{sec}]$ & 0.0020 \\
$\gamma_{9}\left[\mathrm{mmHg} \mathrm{sec} / \mathrm{cm}^{3}\right]$ & 3.9006 & & \\
\hline
\end{tabular}

Table 3: Parameters obtained after optimization. Time-constants $\tau_{i}$ denote the time delay involved with the controlled variables, parameters for gravity denote the max height needed to obtain observed pressure drop, and the length of the time over which the subject stands up. Optimized values for the resistances $\gamma(1)-\gamma(9)$ denote the optimized values for the cerebrovascular resistance $R_{a c p}$. Finally, subscript $k$ gives the steepness of the sigmoid in the set-point functions in equation (15). The scaling factors, subscript $f$, give the scaling for $x_{\min }$ and $x_{\max }$ in equation (15). 


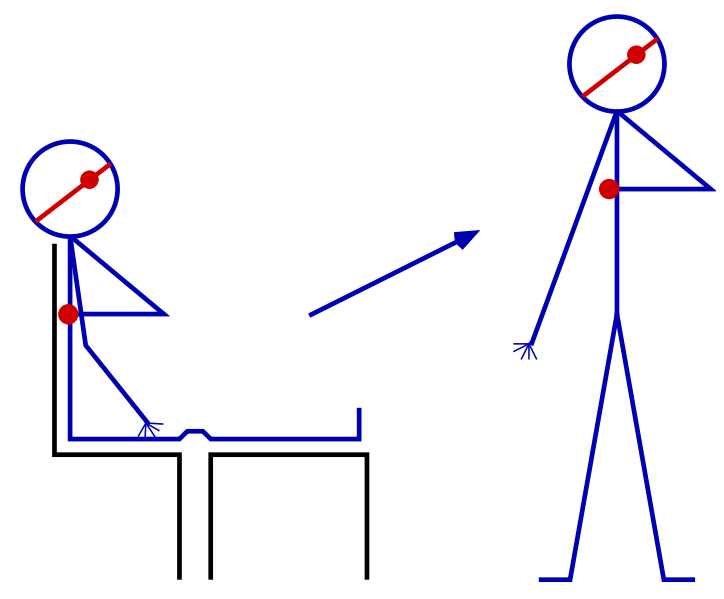

Figure 1: Experimental setup 


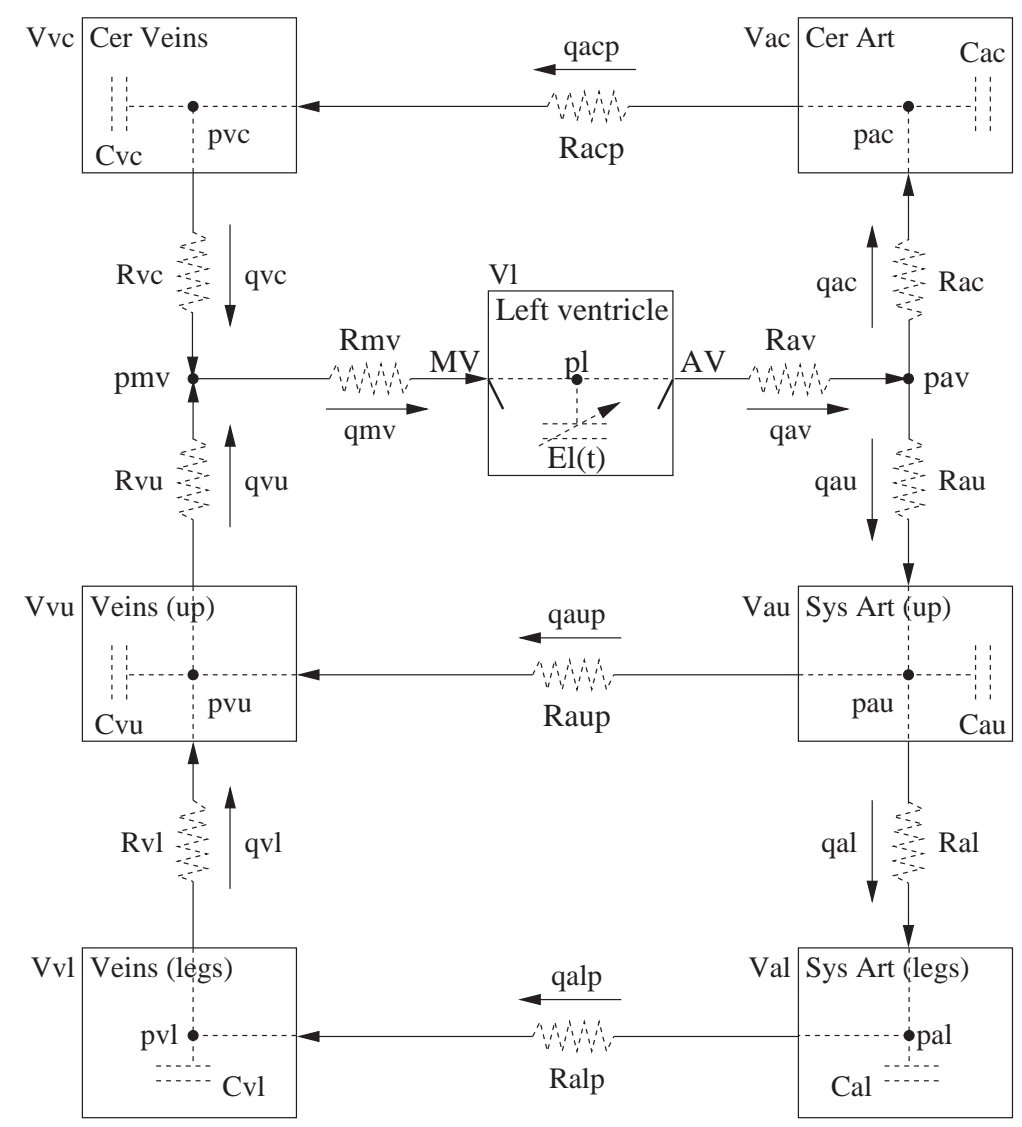

Figure 2: The circuit. Following terminology from electrical circuit theory, resistors $R$ [mmHg $\mathrm{sec} / \mathrm{cm}^{3}$ ] are marked with dashed zig-zag lines. Capacitors $C\left[\mathrm{~cm}^{3} / \mathrm{mmHg}\right]$ representing compliance are marked with dashed parallel lines inside compartments (indicating that they contain volumes), and the aortic and mitral valves are marked with small lines inside the compartment representing the left ventricle. The abbreviations are $l$ left ventricle, $a v$ aortic valve, $m v$ mitral valve, au upper arteries, al lower arteries, aup upper peripheral arteries, alp lower peripheral arteries, $a c$ cerebral arteries, acp peripheral cerebral arteries, $v l$ lower systemic veins, $v u$ upper systemic veins, $v c$ cerebral veins. 

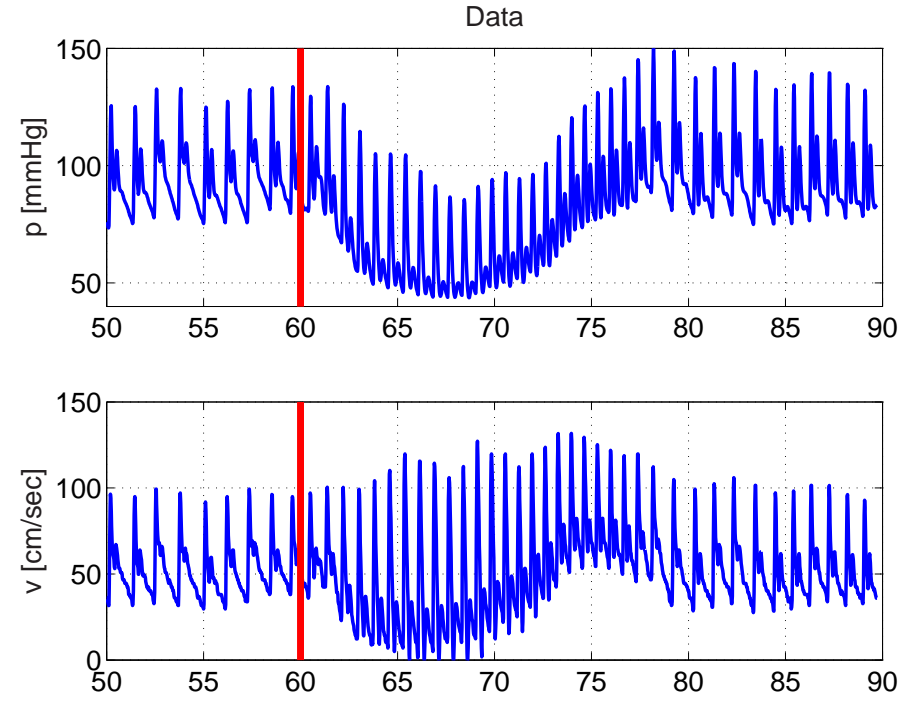

Figure 3: Measurements of cerebral blood flow velocity $v[\mathrm{~cm} / \mathrm{sec}]$ and arterial pressure $p[\mathrm{mmHg}]$. The subject stands up at $t=60 \mathrm{sec}$. 


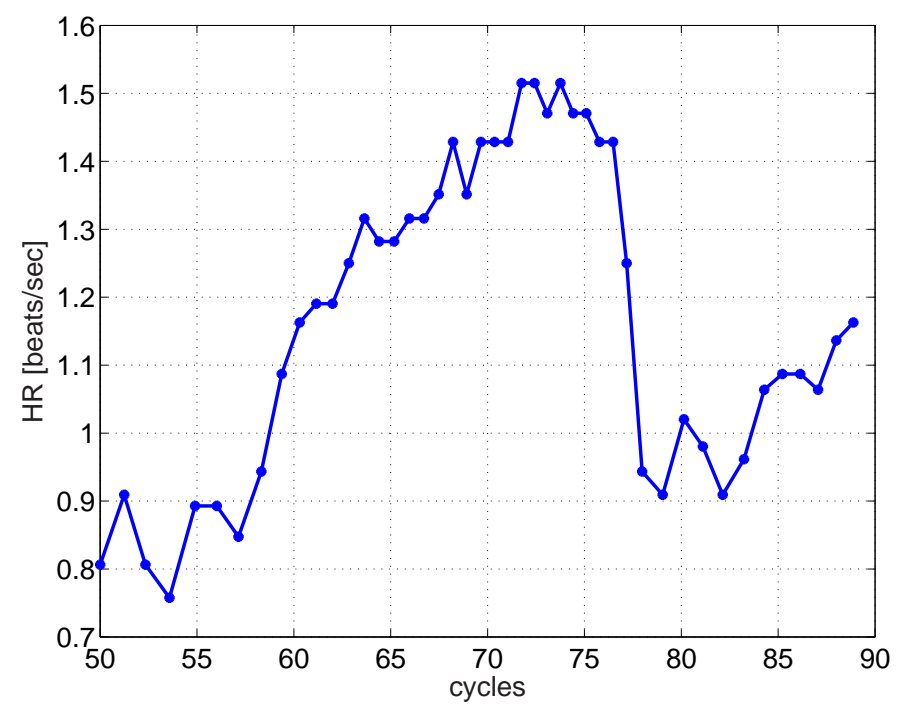

Figure 4: The heart-rate is not controlled but taken from the data. The hear-rate HR [beats/sec] is plotted as a function of time. The $y$-value for each star indicates the value of the heart-rate, the $x$-value indicates the time for onset of that cardiac cycle. 

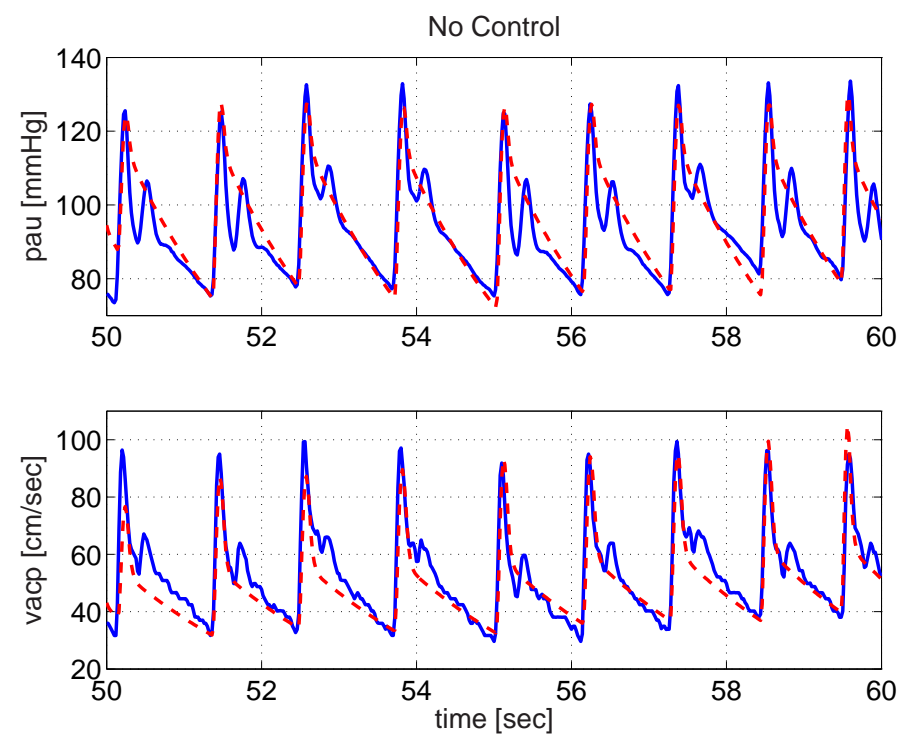

Figure 5: Modeling of cerebral blood flow velocity and arterial pressure $\left(v_{a c}[\mathrm{~cm} / \mathrm{sec}]\right.$ and $p_{a u}$ $[\mathrm{mmHg}]$ on Figure 2). Note on the circuit it is indicated that our model computes flow $\left[\mathrm{cm}^{3} / \mathrm{sec}\right]$ not velocity $[\mathrm{cm} / \mathrm{sec}]$. The velocity shown in this figure is obtained by scaling the flow with a constant factor representing the cross-sectional area of the middle cerebral artery. The dashed line shows the result of our computation and the solid line shows the data. 

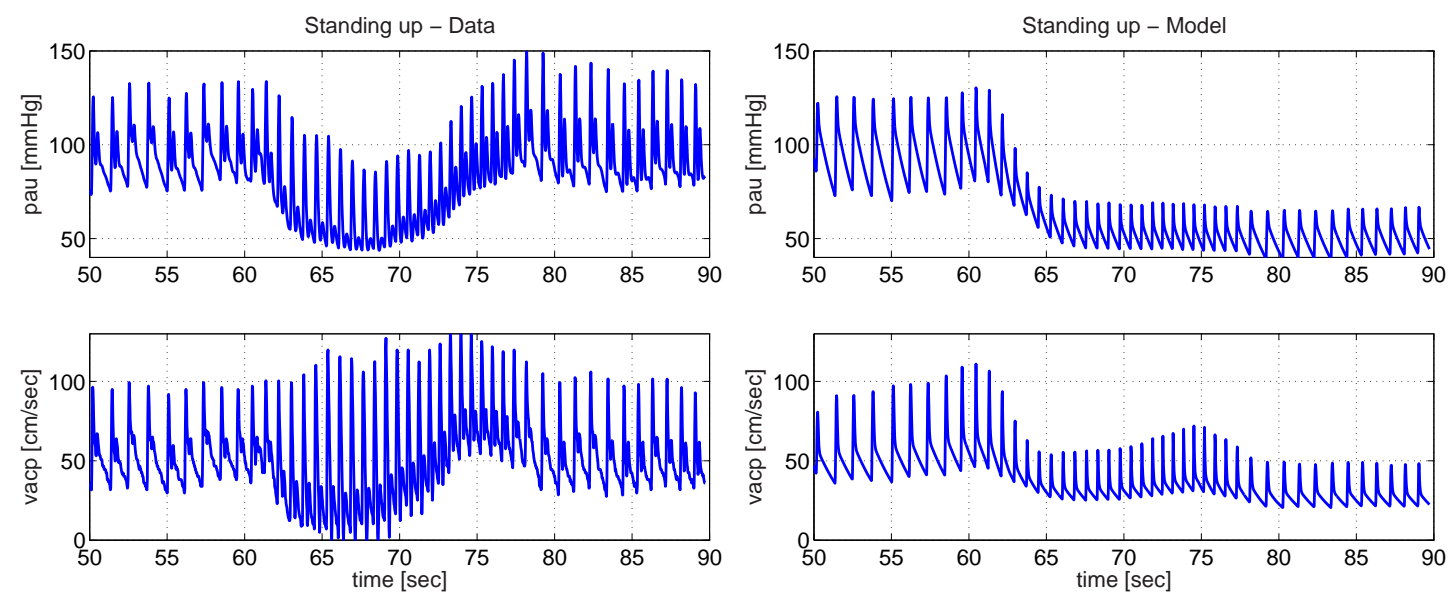

Figure 6: Modeling of cerebral blood flow velocity and arterial pressure $\left(v_{a c}[\mathrm{~cm} / \mathrm{sec}]\right.$ and $p_{a u}$ $[\mathrm{mmHg}]$ on Figure 2). This figure shows the effect of standing up without including control mechanisms. The figure shows that both blood flow velocity and pressure decreases due to the redistribution of volumes obtained by changes in the hydrostatic pressure. 

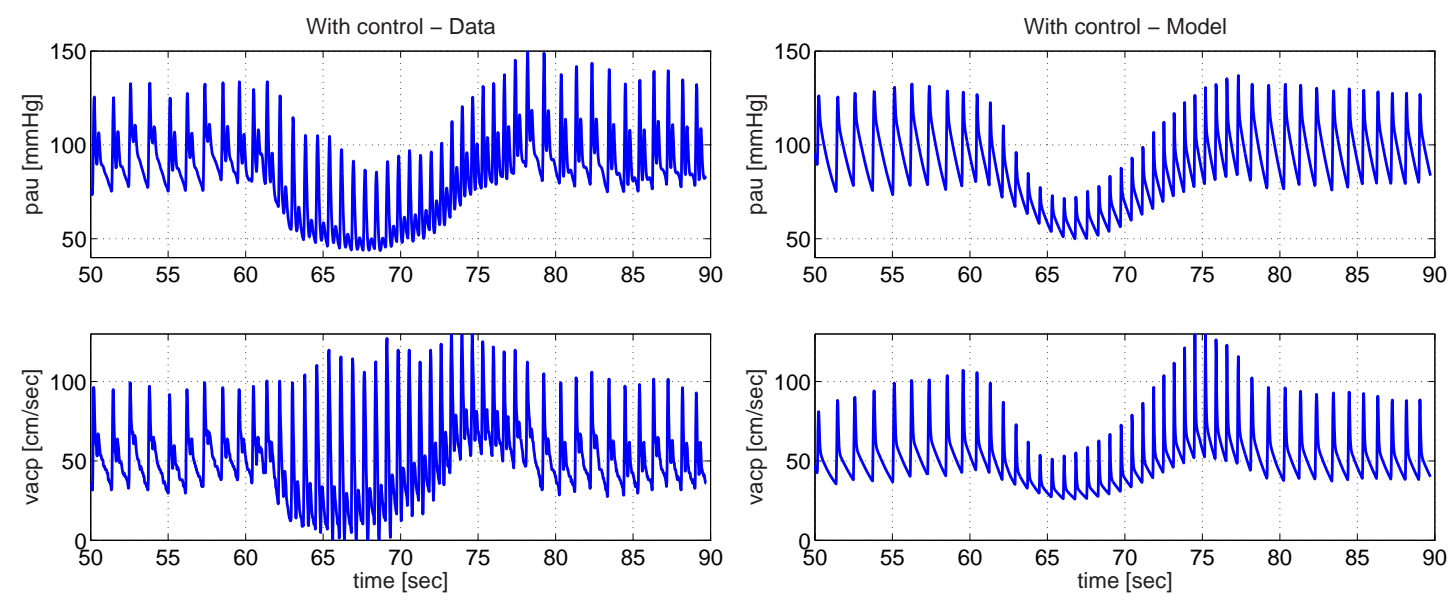

Figure 7: With control, both blood pressure $p[\mathrm{mmHg}]$ and blood flow velocity $v[\mathrm{~cm} / \mathrm{sec}]$ recover to normal levels after the transitional period. 

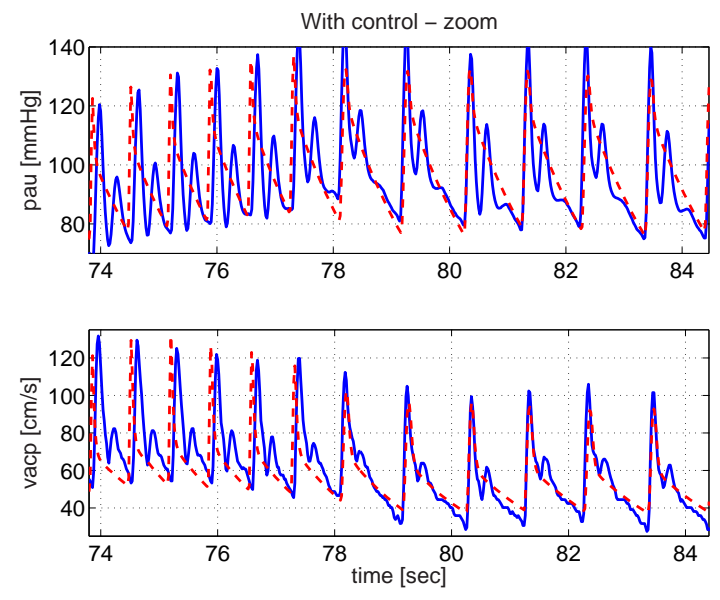

Figure 8: Zoom of the control for the cardiac cycles for $73.8 \leq t \leq 84.5$. The dashed curve shows our computed results and the solid curve shows the data. The results show that even though, the oscillations were too narrow during the transition, we do obtain a very good fit for the end of the regulatory domain. 


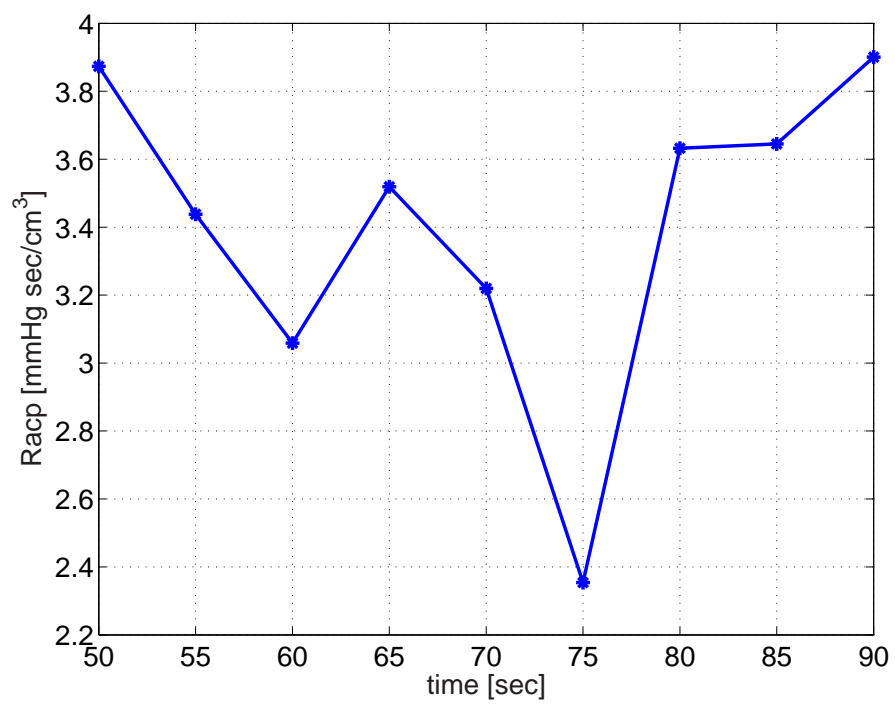

Figure 9: Cerebral vascular resistance $R_{a c p}$. We used 9 values to fit the cerebral vascular resistance to data. Our results show an initial decrease followed by an increase, and then a decrease. We expect that the first increase is a consequence of autonomic regulation, while the second decrease is a result of cerebral autoregulation. 

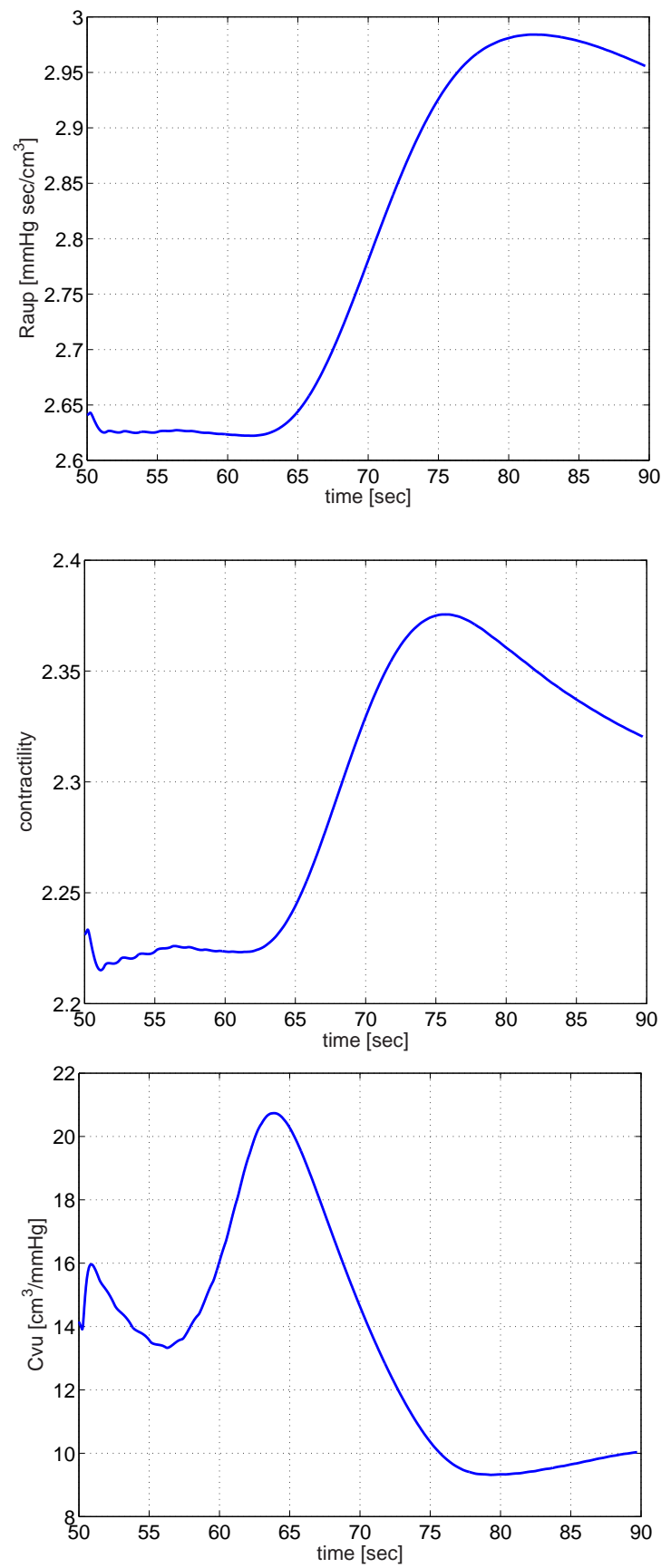

Figure 10: Dynamics of the controlled variables. The figure shows the peripheral resistance in the upper body $R_{a u p}$, the cardiac contractility $c_{v u}$, and the compliance of the veins in the upper body. Other resistances and compliances were regulated similar to the ones shown here. 


\section{References}

[1] Aaslid R, Lindegaard K, Sorteberg W, Nornes H. Cerebral Autoregulation Dynamics in Humans. Stroke 20(1):45-52, 1989.

[2] Aaslid R, Newell DW, Stoos R, Sorteberg W, Lindegaard K. Assessment of cerebral autoregulation dynamics and venous transcranial Doppler recordings in humans. Stroke 22:1148$1154 \mathrm{~m} 1991$.

[3] Aaslid R. Cerebral Hemodynamics. In Transcranial Doppler, Newell DW, Aaslid R. (editors) Raven Press, New York, 49-55, 1992.

[4] Cigada M, Marzorati S, Tredici S, Lapichino G. Cerebral CO2 vasoreactivity by transcranial Doppler ultrasound technique a standardized methodology. Intensive Car Medicine 26(6):792-732, 2000.

[5] Danielsen, M. and Ottesen, J. A Dynamical approach to the baroreceptor regulation of the Cardiovascular system. Proc. 5th Int. Symposium "Symbiosis '97" (Sept 10-12, Brno The Chech Republic), 25-29, 1997.

[6] Danielsen M. Modeling of feedback mechanisms which control the heart function in a view to an implementation in cardiovascular models. PhD Thesis, Roskilde University, Denmark. Text No 358, 1998.

[7] Danielsen M. and Ottesen J.T. Describing the Pumping Heart as a Pressure Source J Theo Biol 212:71-81, 2001.

[8] Guyton AC, Hall JE. Textbook of medical physiology. WB Saunders, Philadelphia, PE, 9th edn, 1996.

[9] Kelley C. Iterative methods for optimization. SIAM, Philadelphia, PE, 1999. 
[10] Lipsitz LA, Mukai S, Hamner J, Gagnon M, and Babikian V. Dynamic regulation of middle cerebral artery blood flow velocity in aging and hypertension. Stroke, 31(8):1897-1903, 2000.

[11] Joyner M, Shephard JT. Autonomic regulation of the ciculation. In Clinical Autonomic Disorders: Evaluation and Management, Low PA (editor). Little, Brown and Company, Boston, MA, pp. 61-81, 1993.

[12] Low PA. The effect of aging on the autonomic nervous system. In Clinical Autonomic Disorders: Evaluation and Management, Low PA (editor). Little, Brown and Company, Boston, MA, pp. 685-700, 1993.

[13] Low PA, Novak V, Spies JM, Petty G. Cerebrovascular regulation in the postural tachycardia syndrome (POTS). Am J Sciences, 317(2),124-133, 1999.

[14] Mulier J.P., Ventricular pressure as a function of volume and flow. PhD Thesis, University of Leuven, Belgium, 1994.

[15] Olufsen MS. Structured tree outflow condition for blood flow in larger systemic arteries, $A m$ J Physiol, 276:H257-H268, 1999.

[16] Olufsen MS, Peskin CS, Larsen J, Nadim A. Derivation and validation of physiologic outflow condition for blood flow in the systemic arteries. Ann Biomed Eng, 28:1281-1299, 2000.

[17] Olufsen MS, Nadim A, and Lipsitz LA. Dynamics of cerebral blood flow regulation explained using a lumped parameter model. Am J Physiol, 282:R611-R622, 2002.

[18] Olufsen MS, Nadim A. On deriving lumped models for blood flow and pressure in the systemic arteries. Proceeding from the 2nd MIT conference of Computational Fluid and Solid Mechanics, KJ Bathe (ed). Elsevier Science, pages 1786-1789, 2003.

[19] Ottesen JT. Modeling of the baroreflex-feedback mechamism with time-delay. J Math Biol, 36:41-63, 1997. 
[20] Ottesen JT. General Compartmental Models of the Cardiovascular System. In Mathematical models in medicine. Eds by Ottesen, J.T. and Danielsen, M. IOS press, 2000, 121-138, 2000.

[21] Ottesen JT. Modelling the dynamical baroreflex-feedback control. In Mathematical and Computer Modelling. Eds by edited by Gyori, I. Vol. 31, no. 4-5, 167-173, 2000.

[22] Ottesen J.T. and Danielsen M. Modeling Ventricular Contraction with heart rate changes. Submitted, 2002.

[23] Panerai RB. Assessment of cerebral pressure autoregulation in humans - a review of measurement methods. Physiol Meas, 19:305-338, 1998.

[24] Quanta Healthcare Solutions, Inc. The Medical Algorithms Project. 02.07 Body Surface Area and 02.08 Estimated Blood Volume Based on Body Surface Area. http://www.medal.org, 2002.

[25] Smith JJ, Kampine JT. Circulatory Physiology, the Essentials. Williams and Wilkins, Baltimore, MD, 3rd edn, 1990.

[26] Strandgaard S, Paulson OB. Cerebral blood flow in untreated and treated hypertension. Neth J Med, 47:180-184, 1995.

[27] Sugawa K., Suga H., and Nakayama K. Instantaneous pressure-volume ration of the left ventricle versus instantaneous force-length relation of papillary muscle. In Cardiovascular System Dynamics. Eds by Baan J., Noordergraaf A. and Raines J. MIT press, Cambridge, MA, 99-105, 1978.

[28] Tiecks FP, Lam AM, Aaslid R, Newell DW. Comparison of static and dynamic cerebral autoregulation measurements. Stroke, 26:1014-1019, 1995.

[29] Traon AP, Costes-Salon MC, Galinier M, Fourcade J, Larrue V. Dynamics of cerebral blood flow autoregulation in hypertensive patients. J Neurol Sci, 195:139-144, 2002. 
[30] Warner HR. The Frequency-dependent Nature of Blood Pressure Regulation by Carotid Sinus Studied with an Electric Analog Circ Res, VI:35-40, 1958. 